\title{
A novel group analysis for functional MRI of the human brain based on a two-threshold correlation (TTC) method
}

\author{
Tibor Auer ${ }^{\mathrm{a}}$, Attila Schwarcz ${ }^{\mathrm{a}, \mathrm{b}, *}$, Tamas Doczi ${ }^{\mathrm{a}}$, \\ Klaus-Dietmar Merboldt ${ }^{\mathrm{c}}$, Jens Frahm ${ }^{\mathrm{c}}$ \\ ${ }^{a}$ Department of Neurosurgery, University of Pécs, H-7624 Pécs, Hungary \\ ${ }^{\mathrm{b}}$ Pécs Diagnostic Institute, University of Pécs, H-7624 Pécs, Hungary \\ ${ }^{\mathrm{c}}$ Biomedizinische NMR Forschungs GmbH am Max-Planck-Institut für biophysikalische \\ Chemie, 37070 Göttingen, Germany
}

Received 13 April 2007; received in revised form 1 August 2007; accepted 19 August 2007

\begin{abstract}
This work presents a new group analysis for functional MRI of human brain activation. The two-threshold correlation (TTC) method determines two statistical thresholds by estimating the noise distribution underlying the summed histogram of correlation coefficients (CC) from all sections and subjects. The probabilistic CC thresholds $(p<0.0001$ for the identification of highly significant activation centers and $p<0.05$ for limiting the iterative addition of directly neighboring voxels to these centers) are applied to the group CC maps for each section. These maps may be reconstructed by taking the maximum (MAX) or mean (MEAN) CC value of all subjects for a particular voxel. Experimental analyses involved functional echo-planar imaging of sequential finger-to-thumb opposition and silent word generation at $3 \mathrm{~T}$ (eight subjects). Preprocessing included motion correction, spatial filtering, and normalization to MNI space. While the results for the TTC MAX approach were very similar to those obtained for a standard SPM analysis, the TTC MEAN approach turned out to be more conservative emphasizing voxels that are activated in most rather than in only a few subjects. The new method is simple, fast, and robust by linking two thresholds in a physiologically meaningful manner. (C) 2007 Elsevier B.V. All rights reserved.
\end{abstract}

Keywords: Functional MRI; Group analysis; SPM

\section{Introduction}

Functional magnetic resonance imaging (fMRI) of human brain activation is a non-invasive method for monitoring hemodynamic responses to a functional challenge in single subjects with high spatial and temporal resolution. However, in view of increasingly complex cognitive paradigms that often involve only subtle differences in neural activity, the response strengths tend to decrease and impose severe challenges for adequate post-processing. A common strategy is therefore to move from single subjects to group analyses and the most widely employed method is statistical parametric mapping (SPM) (Friston et al., 1995). Though elegantly designed for the statistically trained and experienced researcher, a potential drawback of the method is its

\footnotetext{
* Corresponding author at: Department of Neurosurgery, University of Pécs, Rét u. 2, Pécs H-7624, Hungary. Tel.: +36 72535 932; fax: +36 72535931.

E-mail address: attila.schwarcz@aok.pte.hu (A. Schwarcz).
}

complexity. As a consequence, SPM applications require substantial knowledge with the potential risk that the rank-and-file user may be misguided to produce inadequate results.

On the other hand, at the single-subject level, a fMRI data processing method has been established (Baudewig et al., 2003) that emerges as a rather simple data-driven approach originally motivated by detailed experimental observations and physiologic considerations (Kleinschmidt et al., 1995). The method is referred to as two-threshold correlation (TTC) and has evolved during the analysis of several thousands of fMRI data sets acquired in multiple studies of the Göttingen laboratory. It is based on correlation coefficient (CC) maps that are thresholded individually by estimating the noise distribution underlying the distribution (or histogram) of correlation coefficients of the actual fMRI acquisition. In fact, studies without any stimulation (Null experiment) demonstrated that the width of a corresponding CC distribution may be affected by alterations of the hemodynamic responsiveness ('arousal'), respiration, perfusion, flow-induced tissue pulsations, or motions. Because the 
basic form of these distributions is adequately described by a Gaussian curve, true brain activations, that are voxels representing paradigm-associated fMRI signal alterations, may be easily identified: the CC histogram of a fMRI study emerges as the sum of a dominating noise distribution and a second much smaller distribution of activated voxels with high positive (or negative) $\mathrm{CC}$ values. In contrast to methods based on a single threshold, the TTC method employs two probabilistic thresholds for their separation: a high value for the identification of highly significant activations and a lower value for limiting the iterative addition of directly neighboring voxels to these centers. The approach ensures both specificity and sensitivity for defining the spatial extent of significant activation spots.

The aim of this study was to extend the TTC method to group analyses in order to offer an easy-to-use and robust alternative to other more complex post-processing strategies for fMRI. Brain activation was elicited with standard task paradigms and the results were compared to corresponding group analyses with SPM.

\section{Materials and methods}

\subsection{Participants and paradigms}

Eight healthy normal volunteers (male, right-handed, mean age: $31 \pm 4$ years) participated in the study. Handedness was examined by the Edinburgh Inventory Test (Oldfield, 1971). Approval by the Institutional Review Board was obtained and all subjects gave written informed consent before each examination.

Mapping of sensorimotor and language areas was accomplished with use of a block design alternating active and passive periods each lasting for $50 \mathrm{~s}$. The paradigms involved five cycles of unilateral sequential finger-to-thumb opposition (right hand, frequency 1-2 Hz) and motor rest (Baraldi et al., 1999) and seven cycles of silent word generation and rest (Maldjian et al., 2002; Woermann et al., 2003).

\section{2. $M R I$}

MRI was conducted at $2.89 \mathrm{~T}$ (Siemens Tim Trio, Erlangen, Germany). Excitation was performed with the body coil, while signal reception was achieved with an 8-channel head coil. Functional MRI involved a standard EPI sequence $\left(\mathrm{TR} / \mathrm{TE}=2500 / 36 \mathrm{~ms}\right.$, flip angle $\left.80^{\circ}\right)$ with an isotropic spatial resolution of $3 \mathrm{~mm}$ ( 24 sections). Motion correction was performed as offered by the manufacturer. All images were subjected to a spatial Gaussian filter of $5 \mathrm{~mm}$ full width at half maximum and normalized to MNI space (Collins, 1994). Both SPM and TTC post-processing steps were applied to these normalized and spatially smoothed images using MatLab 6.5 software.

\subsection{SPM group analysis}

Group analyses were performed with SPM5 (Friston et al., 1995). We have specified our fixed-effect model based on default settings of SPM with the eight subjects together using first-level analysis: Basic hemodynamic response function, canonical hrf, high-pass filter: cutoff of $128 \mathrm{~s}$; global calculation: mean voxel value; grand mean scaling: session specific; global normalization: none. AR(1) model was used to account for substantial autocorrelations in fMRI data. We used contrasts "1 1111111111 " for activation and " $-1-1-1-1-1-1-1-1$ " for deactivation for the eight subjects respectively. A statistical threshold of $p<0.05$ with FWE correction (Nichols and Hayasaka, 2003) was applied resulting in $t=4.66$ for silent word generation and $t=4.62$ for sequential finger opposition tasks, respectively. Activation and deactivation are shown on the same maps derived from $t$ score maps produced by SPM5 software.

\subsection{TTC group analysis}

Similar to the established single-subject TTC analysis (Kleinschmidt et al., 1995; Baudewig et al., 2003), correlation coefficient maps were calculated for each subject with use of a simple boxcar reference function derived from the task protocol and shifted by $5 \mathrm{~s}$ to account for the delayed hemodynamic
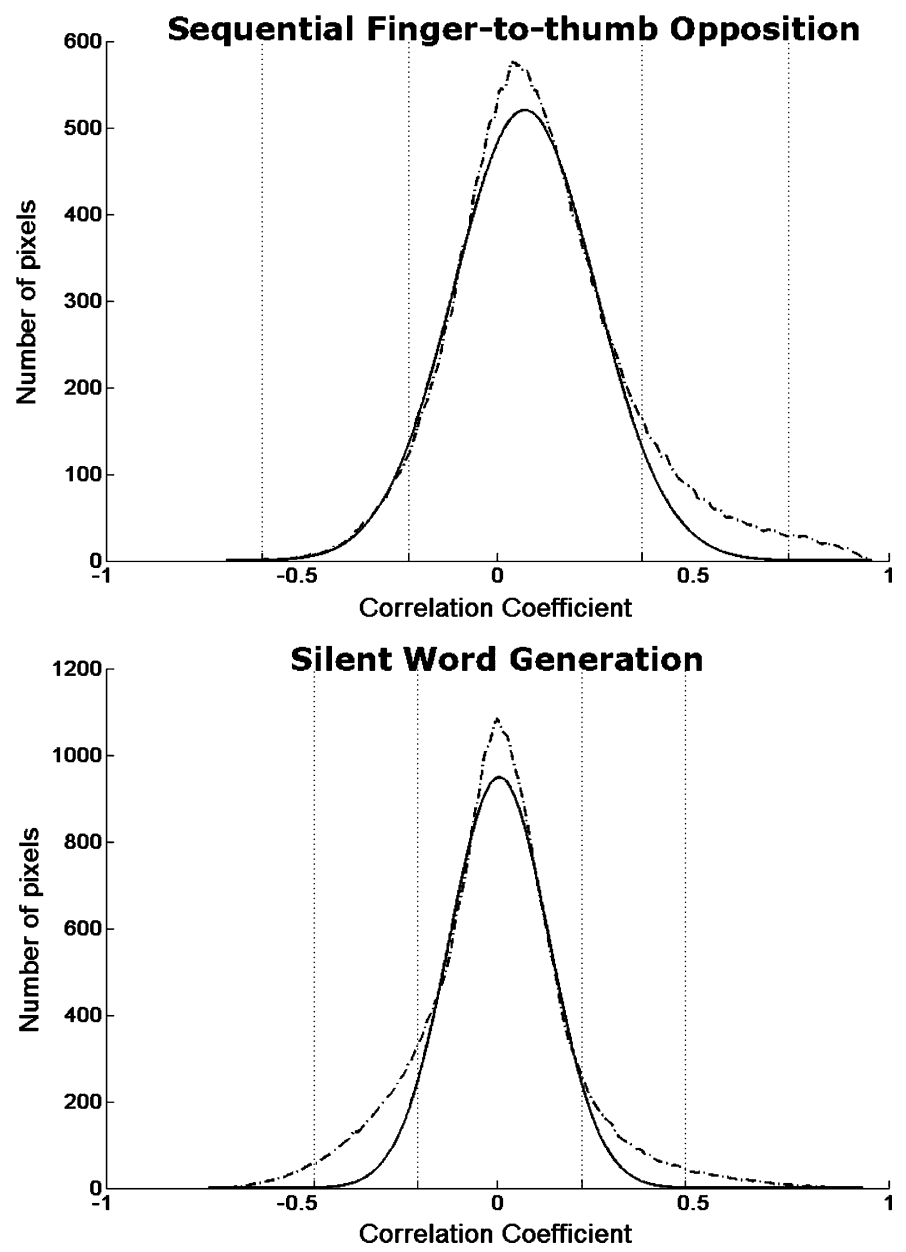

Fig. 1. Group distributions of $\mathrm{CC}$ values from all subjects and sections (dotted line) for sequential finger-to-thumb opposition and silent word generation. The fit of a Gaussian function to the central portion of the $\mathrm{CC}$ distributions (solid line) defines the underlying noise distribution. It reveals deviations for high positive and high negative $\mathrm{CC}$ values indicating activation and deactivation, respectively. Vertical lines refer to the 99.99 and 95 percentile ranks of the noise distributions. 
response (Bandettini et al., 1993). Two CC thresholds for the group analysis were determined by fitting a Gaussian function to the central portion of the group distribution of $\mathrm{CC}$ values from all subjects and sections (i.e., from all individual $\mathrm{CC}$ maps). This part of the $\mathrm{CC}$ distribution has low $\mathrm{CC}$ values that do not refer to stimulus-correlated events. It may therefore be regarded as a representation of the noise distribution underlying the $\mathrm{CC}$ distribution of the actual experiment (Kleinschmidt et al., 1995).

With use of the estimated noise distribution the actual $\mathrm{CC}$ distribution is rescaled into percentile ranks of the noise distribution. Thus, the upper threshold chosen in the TTC analysis corresponds to the CC that matches the $99.99 \%$ ( $p$ value $=0.0001$ ) percentile rank of the noise distribution. Voxels exceeding this value become automatically accepted as activated-they form the highly significant activation centers. Directly neighboring voxels are iteratively added to improve the area delineation around an activation center as long as their CC values exceed a lower threshold corresponding to the $95 \%$ ( $p$ value $=0.05$ ) percentile rank of the noise distribution.

After establishment of the $\mathrm{CC}$ thresholds for all sections, a voxelwise determination of respective group $\mathrm{CC}$ maps is achieved in two different ways: by a selection of either the maximum $\mathrm{CC}$ value (MAX) from the range of $\mathrm{CC}$ values of the individual $\mathrm{CC}$ maps or by calculating a mean $\mathrm{CC}$ value (MEAN) for each individual voxel. The resulting group $\mathrm{CC}$ maps are then analyzed with the previously determined thresholds in the prescribed manner to yield a group activation map for each section-similar to the procedure for a single-subject
TTC analysis. Activations and deactivations are visualized on the same map. In order to characterize negative correlations, the group maps are reconstructed with use of the minimum or mean negative $\mathrm{CC}$ values for the MAX and MEAN approach, respectively.

\section{Results}

Fig. 1 shows the group distributions of $\mathrm{CC}$ values from all subjects and sections for the two tasks studied. When comparing the actual distribution and the estimated noise distribution, active finger movements were mainly characterized by deviations in the positive $\mathrm{CC}$ range indicating activations. The word generation paradigm revealed deviations for both high positive and negative $\mathrm{CC}$ values that correspond to activated and deactivated voxels, respectively.

Figs. 2 and 3 summarize group activation maps obtained by SPM and TTC for finger movements and word generation (four sections each), respectively. In either case, the first row shows SPM group maps, while the bottom two rows refer to the group maps obtained with TTC MAX and TTC MEAN, respectively. All post-processing methods identified the sensorimotor and supplementary motor areas for the motor task as wells as the Broca region for the word generation paradigm. The locations and the extent of activations/deactivations were similar for the maps analyzed with SPM and TTC MAX, whereas activations/deactivations appeared much more restricted when using the TTC MEAN approach. A brief quantitative comparison of

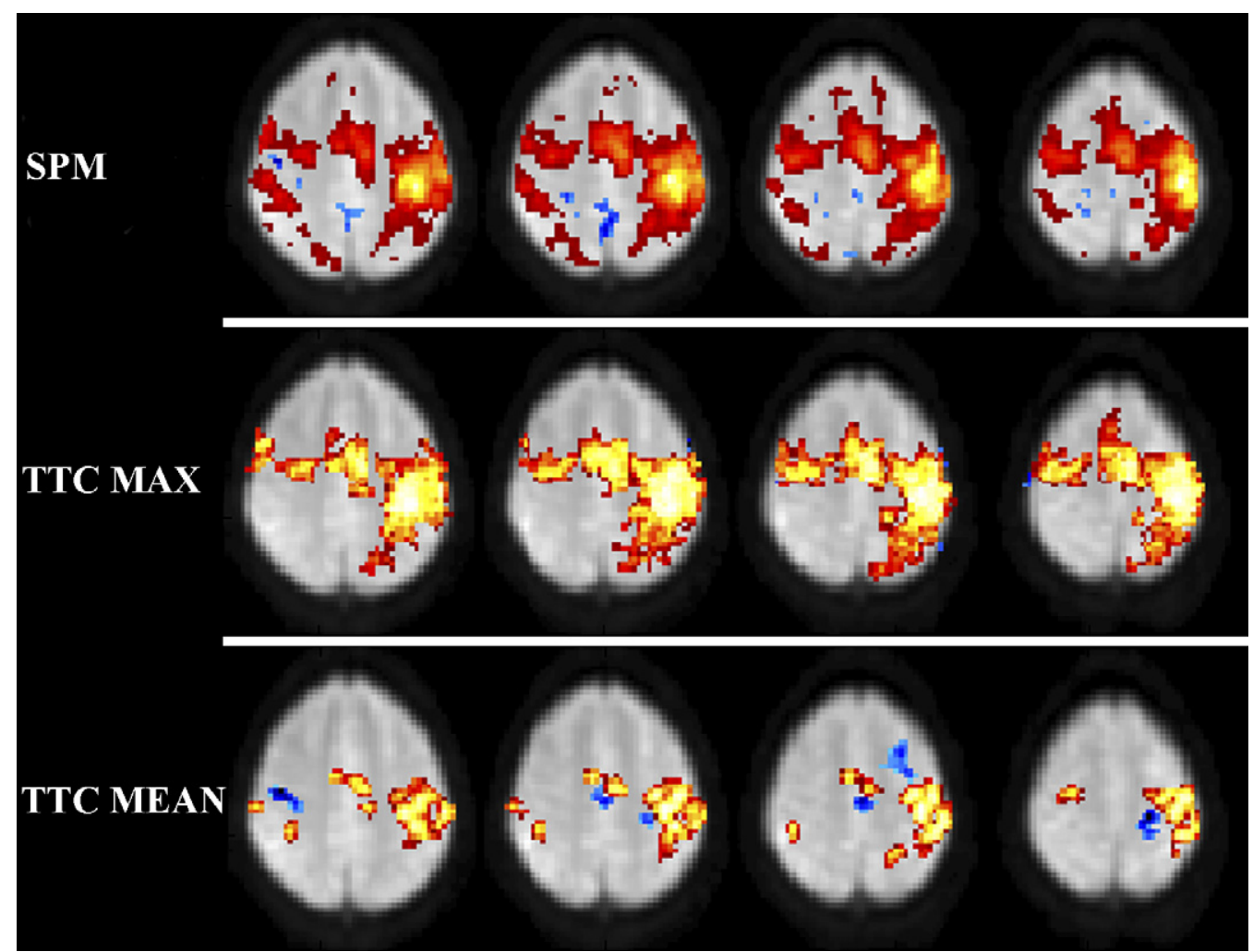

Fig. 2. Group activation maps obtained for sequential finger-to-thumb opposition of the right hand. Group maps are calculated with SPM, TCC MAX and TTC MEAN approaches. Activations and deactivations are indicated by red and blue colors, respectively. 


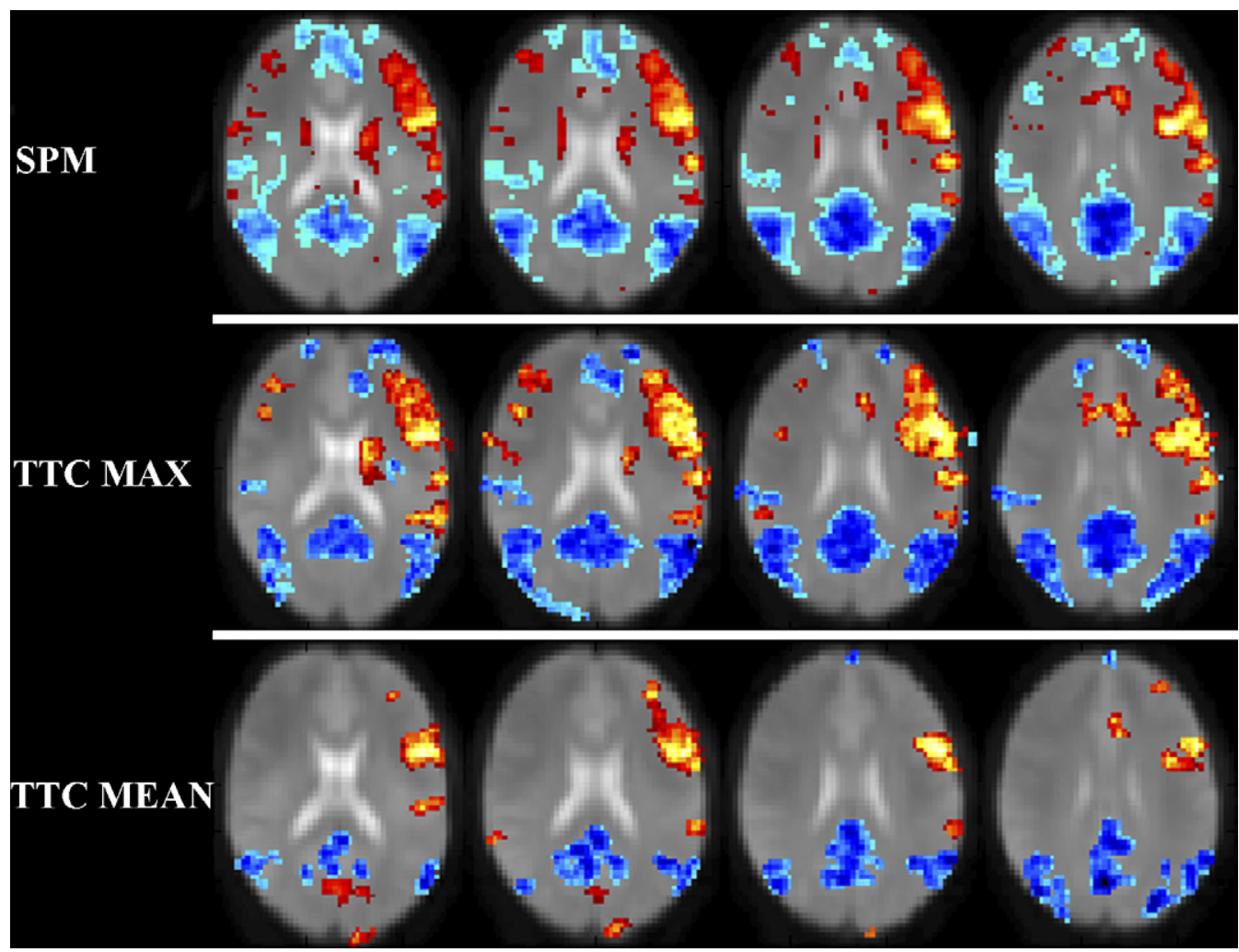

Fig. 3. Group activation maps obtained for silent word generation. The order and color coding of maps is identical to that of Fig. 2.

activated voxels obtained for silent word generation with use of SPM, TTC MAX, and TTC MEAN is given in Table 1 and supports the qualitative findings from the images.

\section{Discussion}

Despite a widespread acceptance and availability of SPM, the analysis of fMRI data sets remains a field open for improvements. The idea of this work is not to design a new mathematical framework, but to describe a simple and transparent technique.

While most of the proposed analysis strategies rely on a single statistical threshold (sometimes supplemented by a cluster threshold), the key of the TTC method is a combination of two thresholds in a physiologically meaningful manner. In more detail, the resulting advantageous features may be explained as follows. First of all, in the absence of a functional challenge, correlation analyses have been demonstrated to result in a symmetric distribution of $\mathrm{CC}$ values (centered around zero) which may be characterized by a Gaussian function (Kleinschmidt et al., 1995). This noise distribution reflects physiologic fluctuations and/or 'resting state' brain activity of the subject and as

Table 1

Total activations and deactivations obtained for silent word generation with SPM, TTC MAX, and TTC MEAN

\begin{tabular}{llll}
\hline & SPM & TTC MAX & TTC MEAN \\
\hline \# Activated voxels & 4326 & 5292 & 2449 \\
\# Deactivated voxels & 7268 & 6439 & 2814 \\
\hline
\end{tabular}

such may vary in broadness from experiment to experiment. It is therefore mandatory to estimate the noise distribution for the actual fMRI acquisition to be analyzed. This is accomplished by restricting the fit to the central portion of the actual CC distribution which contains only low $\mathrm{CC}$ values of voxels not engaged in task performance. This procedure ensures substantial robustness of the TTC analysis and certainly facilitates intertrial comparisons by removing systemic alterations not related to the interrogated functional state.

Secondly, the actual CC distribution is rescaled in percentile ranks of the noise distribution. This step is followed by an automatic definition of two statistical thresholds which are tightly linked by neighborhood principles when used for calculation of an activation map. While the upper threshold focuses on the identification of highly significant activations, the lower threshold is only used to better delineate their spatial extent rather than to incorporate new activations of low significance. This strategy may be characterized as defining the mountain around its peak. It yields robust statistical parametric maps of activated voxels with both high specificity and sensitivity under the assumption that voxels within a truly activated area bear a tight topographic relationship to a smaller number of voxels which exceed a threshold related to a very low type-one error probability (false positives). The method should not be mistaken for a 'cluster analysis' that relies on assumptions about the cluster size in relation to the image resolution. Specifically, the spatial response delineation used here does not exclude small - even single voxel - activations which are necessarily omitted in a conventional cluster analysis. 
Two different group CC maps have been tested (bottom rows of Figs. 2 and 3). A maximum sensitivity is obtained for the TTC MAX approach which is based on group CC maps that incorporate the maximum (or minimum) $\mathrm{CC}$ values observed in the entire group of subjects. The general appearance of these maps is similar to what may be obtained with a standard SPM analysis (top row of Figs. 2 and 3). Conversely, when using the mean value rather than the extreme $\mathrm{CC}$ values, the TTC MEAN results appear more conservative and demonstrate activated (or deactivated) clusters in a very restrictive manner. Taken together, the TTC MAX approach may include voxels that are active in only few of the subjects (or even a single subject with a single high CC value), whereas the TTC MEAN approach relies on activations more common to the group (or in most of the subjects yielding a high mean CC value). In this sense the TTC MEAN method successfully eliminates false positive activations.

In conclusion, the proposed TTC group analysis for fMRI appears to be advantageous in several respects: (i) it is simple, transparent, and fast, (ii) it is robust with respect to physiologic variability by estimating the noise distribution and respective thresholds from the actual experiment, and (iii) it may minimize the problem of false positive activations by combining two thresholds for the identification of activations and by taking the mean of individual $\mathrm{CC}$ values for the respective group analysis.

\section{References}

Bandettini PA, Jesmanowicz A, Wong EC, Hyde JS. Processing strategies for time-course data sets in functional MRI of the human brain. Magn Reson Med 1993;30:161-73.

Baraldi P, Porro CA, Serafini M, Pagnoni G, Murari C, Corazza R, et al. Bilateral representation of sequential finger movements in human cortical areas. Neurosci Lett 1999;269:95-8.

Baudewig J, Dechent P, Merboldt KD, Frahm J. Thresholding in correlation analyses of magnetic resonance functional neuroimaging. Magn Reson Imaging 2003;21:1121-30.

Collins, DL. 3D Model-based segmentation of individual brain structures from magnetic resonance imaging data. Thesis. Canada: McGill University; 1994.

Friston KJ, Holmes AP, Worsley KP, Poline JP, Frith CD, Frackowiak RSJ. Statistical parametric maps in functional imaging: a general linear approach. Hum Brain Map 1995;2:189-210.

Kleinschmidt A, Requardt M, Merboldt KD, Frahm J. On the use of temporal correlation coefficients for magnetic resonance mapping of functional brain activation. Individualized thresholds and spatial response delineation. Intern J Imag Syst Technol 1995;6:238-44.

Maldjian JA, Laurienti PJ, Driskill L, Burdette JH. Multiple reproducibility indices for evaluation of cognitive functional MR imaging paradigms. AJNR 2002;23:1030-7.

Nichols T, Hayasaka S. Controlling the familywise error rate in functional neuroimaging: a comparative review. Stat Methods Med Res 2003;12:419-46.

Oldfield RC. The assessment and analysis of handedness: the Edinburgh Inventory. Neuropsychologia 1971;9:97-113.

Woermann FG, Jokeit H, Luerding R, Freitag H, Schulz R, Guertler S, et al. Language lateralization by Wada test and fMRI in 100 patients with epilepsy. Neurology 2003;61:699-701. 